\title{
Retrograde Jejuno-Jejunal Intussusception after Total Gastrectomy
}

\author{
Akira Yoneda ${ }^{a, b}$ Yukio Kamohara ${ }^{a}$ Ken Taniguchib \\ Junpei Maeda ${ }^{a}$ Arifumi Akashi $^{\mathrm{a}} K_{\text {Keiji Inoue }}{ }^{\mathrm{a}}$ \\ Norihiro Kohara $^{\mathrm{a}}$ Akimi Miyata $^{\mathrm{a}}$ Takashi Kanematsu $^{\mathrm{b}}$ \\ Department of Surgery, ${ }^{a}$ Nagasaki Municipal Hospital and ${ }^{b}$ Nagasaki University \\ Graduate School of Biomedical Sciences, Nagasaki, Japan
}

\section{Key Words}

Retrograde intussusception · Jejuno-jejunal intussusception · Post gastrectomy complication

\begin{abstract}
An eighty-year-old female was transferred to the hospital after experiencing abdominal pain and nausea. She had had a history of total gastrectomy for gastric cancer 14 years previously. Abdominal X-ray revealed a localized expansion of the small bowel. Computed tomography revealed a mass with a lamellar structure in a concentric circle. With a tentative diagnosis of small bowel obstruction due to intussusception, she underwent emergency operation. Laparotomy revealed a retrograde jejuno-jejunal intussusception. Bowel resection was performed due to the severe ischemic damage. All reported intussusception cases after total gastrectomy displayed retrograde characteristics and could occur both during the early and late period after surgery. It is important to consider the possibility of intussusception for patients presenting with acute abdomen who have previously undergone gastric resection.
\end{abstract}

\section{Introduction}

Among the complications that arise following gastrectomy, such as reflux esophagitis, anastomotic ulcer, dumping syndrome or adhesional ileus, intussusception of the small bowel is a rare but severe complication which frequently requires emergency operation. This report describes the case of a patient who presented with retrograde jejuno-jejunal intussusception occurring 14 years after total gastrectomy. 


\section{Case Report}

An eighty-year-old woman was transferred to the hospital complaining of abdominal pain and nausea. She had undergone total gastrectomy and reconstruction 14 years earlier which was performed with a modified Billroth II method for gastric cancer.

A mobile mass was palpated in the left lower quadrant and tenderness was present at the epigastrium. Laboratory analysis showed moderate leukocytosis and minor anemia. Biochemical findings, electrolyte findings and tumor markers were normal. Abdominal X-ray revealed a localized

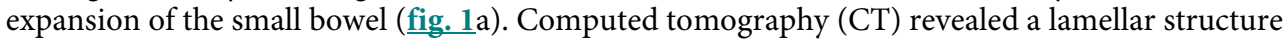
arranged in a concentric circle; however, no mass lesion was found (fig. 1b).

With a tentative diagnosis of small bowel obstruction due to intussusception, she underwent emergency laparotomy. The surgery revealed a jejuno-jejunal intussusception. The distal (anal) intestine had been engulfed by the oral end in the region of the jejunojejunostomy (Braun's anastomosis) (fig. 2). Necrosis of the small intestine was evident after manual reintegration. Bowel resection was therefore performed. The postoperative course was uneventful and no recurrence of the intussusception has been observed during follow-up.

\section{Discussion}

Intussusception after gastrectomy is a comparatively rare disorder. Intussusception occurs in only $0.07-2.1 \%$ of individuals who undergo gastrectomy [1]. However, $87.7 \%$ of intussusceptions following abdominal surgery occur after gastrectomy [1]. Intussusception can be categorized as anterograde (normal peristaltic) or as retrograde (reverse peristaltic) intussusception. Many anterograde intussusceptions occur during the early period following gastrectomy, while the retrograde cases generally arise later [2]. Many cases of intussusception are associated with a Billroth II reconstruction I or the Roux-en-Y method; those associated with a Billroth I reconstruction are rare [3]. Intussusceptions are frequently observed in the region of the anastomotic entrance, including Braun's anastomosis [4].

A review of the literature revealed 20 cases of intussusception occurring after total gastrectomy, including the current case (table 1). A Roux-en-Y reconstruction (the double tract method is included) was reported more frequently (12 cases) than reconstruction using the Billroth II method (including the modified Billroth II, 7 cases). The interval between total gastrectomy and intussusception was more than one year (up to 21 years) in most of the cases; however, some patients experienced intussusception only 6,16 , or 23 days after surgery, thus suggesting that it can occur during both the early and late period. The locus of the intussusception was near the anastomotic region (Roux-en-Y anastomosis and Braun's anastomosis) in all cases. Enterectomy was performed in the majority of cases, but 6 cases required only reintegration.

All intussusceptions after total gastrectomy were characterized as retrograde. A retrograde peristalsis is a normal phenomenon in the small bowel and is often reported among the pathogenic factors of jejuno-jejunal intussusception. Antiperistalsis could be favored by the segmentary motor activity of the small bowel in response to hyperacidity, which is possible after gastrectomy [5]. Various causes of retrograde intussusception are suggested with regard to functional factors, such as (1) helminth aberration and adhesion bending, (2) anastomotic hypersize, (3) excessive behavior of the efferent loop, and (4) intestinal convulsion [1]. These factors affect each other and/or overlap, thus leading to the development of intussusception. Gastrectomy might cause many pathognomonic factors associated with the formation of intussusception, although it was not possible to determine the definitive cause in the current case. 
Echography and CT are extremely useful for the diagnosis of this condition. A target sign or multiple concentric rings are the characteristic findings [6]. There is a report of a jejunal retrograde intussusception to the remaining stomach which was observed using an upper gastrointestinal endoscope [7]. Most of the cases require emergency surgery because of circulatory disturbances in the incarcerated bowel $[5,8]$, whereas the postoperative course is usually good and a recurrence of this condition is quite rare. In patients who do not experience bowel necrosis, some adjuvant modifications are performed to prevent recurrence, such as conversion from a Billroth I to a Billroth I, a chorion suture between the afferent and efferent loop, and so on $[9,10]$. However, the long-term results of these modifications remain unclear.

\section{Conclusion}

This report documents a recent case with retrograde jejuno-jejunal intussusception after total gastrectomy. The early diagnosis of a jejuno-jejunal intussusception is crucial since it requires immediate surgical treatment in many cases, often including an enterectomy. Therefore, it is important to consider intussusception as a post gastrectomy complication both immediately after surgery and during the long-term follow-up.

\section{Acknowledgement}

This works was supported in part by a Nagasaki University President's Fund Grant.

Table 1. Jejuno-jejunal intussusception following total gastrectomy: summary of cases reported in the literature

\begin{tabular}{|c|c|c|c|c|c|c|c|c|c|}
\hline No. & Author & Year & $\begin{array}{l}\text { Age/ } \\
\operatorname{sex}\end{array}$ & Disease & $\begin{array}{l}\text { Reconstruc- } \\
\text { tive method }\end{array}$ & $\begin{array}{l}\text { Interval } \\
\text { since } \\
\text { gastrectomy }\end{array}$ & $\begin{array}{l}\text { Locus of } \\
\text { intussusception }\end{array}$ & Form & $\begin{array}{l}\text { Treat- } \\
\text { ment }\end{array}$ \\
\hline 1 & $\begin{array}{l}\text { Davey } \\
{[11]}\end{array}$ & 1954 & $63 / \mathrm{M}$ & $\begin{array}{l}\text { gastric } \\
\text { cancer }\end{array}$ & Roux-en-Y & 3 years & $\begin{array}{l}? \mathrm{~cm} \text { more anal } \\
\text { from Braun's anastomotic } \\
\text { region }\end{array}$ & $\begin{array}{l}\text { retro- } \\
\text { grade }\end{array}$ & $\begin{array}{l}\text { no ope- } \\
\text { ration, } \\
\text { died }\end{array}$ \\
\hline 2 & Nishi & 1965 & $48 / \mathrm{F}$ & $\begin{array}{l}\text { gastric } \\
\text { cancer }\end{array}$ & Roux-en-Y & 23 days & $\begin{array}{l}8 \mathrm{~cm} \text { more oral } \\
\text { from R-Y anastomotic } \\
\text { region }\end{array}$ & $\begin{array}{l}\text { retro- } \\
\text { grade }\end{array}$ & resection \\
\hline 3 & $\begin{array}{l}\text { Kato } \\
{[12]}\end{array}$ & 1965 & $65 / \mathrm{M}$ & $\begin{array}{l}\text { gastric } \\
\text { cancer }\end{array}$ & Roux-en-Y & 6 days & $\begin{array}{l}10 \mathrm{~cm} \text { more anal from R-Y } \\
\text { anastomotic region }\end{array}$ & $\begin{array}{l}\text { retro- } \\
\text { grade }\end{array}$ & $\begin{array}{l}\text { reintegra- } \\
\text { tion }\end{array}$ \\
\hline 4 & $\begin{array}{l}\text { Freeman } \\
{[10]}\end{array}$ & 1966 & $40 / \mathrm{M}$ & sarcoma & Roux-en-Y & 5 years & $\begin{array}{l}? \mathrm{~cm} \text { more anal from R-Y } \\
\text { anastomotic region }\end{array}$ & $\begin{array}{l}\text { retro- } \\
\text { grade }\end{array}$ & resection \\
\hline 5 & $\begin{array}{l}\text { Christeas } \\
{[13]}\end{array}$ & 1968 & $71 / \mathrm{M}$ & $\begin{array}{l}\text { gastric } \\
\text { ulcer }\end{array}$ & Billroth II & 6 years & $\begin{array}{l}? \mathrm{~cm} \text { more anal from } \\
\text { Braun's anastomotic region }\end{array}$ & $\begin{array}{l}\text { retro- } \\
\text { grade }\end{array}$ & resection \\
\hline 6 & $\begin{array}{l}\text { Hanyu } \\
{[14]}\end{array}$ & 1984 & $39 / \mathrm{F}$ & $\begin{array}{l}\text { gastric } \\
\text { cancer }\end{array}$ & Roux-en-Y & 16 days & $\begin{array}{l}3 \mathrm{~cm} \text { more anal from R-Y } \\
\text { anastomotic region }\end{array}$ & $\begin{array}{l}\text { retro- } \\
\text { grade }\end{array}$ & $\begin{array}{l}\text { reintegra- } \\
\text { tion }\end{array}$ \\
\hline 7 & $\begin{array}{l}\text { Ogata } \\
{[15]} \\
\end{array}$ & 1988 & $59 / \mathrm{M}$ & $\begin{array}{l}\text { gastric } \\
\text { cancer }\end{array}$ & Billroth II & 12 years & $\begin{array}{l}20 \mathrm{~cm} \text { more anal from } \\
\text { Braun's anastomotic region }\end{array}$ & $\begin{array}{l}\text { retro- } \\
\text { grade }\end{array}$ & resection \\
\hline 8 & $\begin{array}{l}\text { Hwang } \\
{[16]}\end{array}$ & 1990 & $59 / \mathrm{M}$ & $\begin{array}{l}\text { gastric } \\
\text { ulcer }\end{array}$ & Billroth II & 10 years & $\begin{array}{l}5 \mathrm{~cm} \text { more anal from } \\
\text { Braun's anastomotic region }\end{array}$ & $\begin{array}{l}\text { retro- } \\
\text { grade }\end{array}$ & resection \\
\hline 9 & $\begin{array}{l}\text { Suganuma } \\
{[17]}\end{array}$ & 1992 & $52 / \mathrm{M}$ & $\begin{array}{l}\text { gastric } \\
\text { cancer }\end{array}$ & Billroth II & 4 years & $\begin{array}{l}30 \mathrm{~cm} \text { more anal from } \\
\text { Braun's anastomotic region }\end{array}$ & $\begin{array}{l}\text { retro- } \\
\text { grade }\end{array}$ & resection \\
\hline$\overline{10}$ & $\begin{array}{l}\text { Hashimoto } \\
{[18]}\end{array}$ & 1993 & $61 / \mathrm{F}$ & $\begin{array}{l}\text { gastric } \\
\text { cancer }\end{array}$ & Roux-en-Y & 10 years & $\begin{array}{l}15 \mathrm{~cm} \text { more anal from R-Y } \\
\text { anastomotic region }\end{array}$ & $\begin{array}{l}\text { retro- } \\
\text { grade }\end{array}$ & $\begin{array}{l}\text { reintegra- } \\
\text { tion }\end{array}$ \\
\hline 11 & $\begin{array}{l}\text { Narishima } \\
{[19]}\end{array}$ & 1994 & $58 / \mathrm{F}$ & sarcoma & Roux-en-Y & 1 year & $\begin{array}{l}10 \mathrm{~cm} \text { more anal from R-Y } \\
\text { anastomotic region }\end{array}$ & $\begin{array}{l}\text { retro- } \\
\text { grade }\end{array}$ & resection \\
\hline
\end{tabular}




\begin{tabular}{|c|c|c|c|c|c|c|c|c|c|}
\hline 12 & $\begin{array}{l}\text { Goto } \\
{[20]}\end{array}$ & 1999 & $75 / \mathrm{M}$ & $\begin{array}{l}\text { esophageal } \\
\text { cancer }\end{array}$ & Roux-en-Y & 9 years & $\begin{array}{l}20 \mathrm{~cm} \text { more anal from } \mathrm{R}-\mathrm{Y} \\
\text { anastomotic region }\end{array}$ & $\begin{array}{l}\text { retro- } \\
\text { grade }\end{array}$ & $\begin{array}{l}\text { reintegra- } \\
\text { tion }\end{array}$ \\
\hline 13 & $\begin{array}{l}\text { Yoshioka } \\
{[21]}\end{array}$ & 1999 & $71 / \mathrm{M}$ & $\begin{array}{l}\text { gastric } \\
\text { cancer }\end{array}$ & Billroth II & 11 years & $\begin{array}{l}5 \mathrm{~cm} \text { more anal from } \\
\text { Braun's anastomotic region }\end{array}$ & $\begin{array}{l}\text { retro- } \\
\text { grade }\end{array}$ & resection \\
\hline$\overline{14}$ & $\begin{array}{l}\text { Sumi } \\
{[22]}\end{array}$ & 2000 & $82 / \mathrm{M}$ & $\begin{array}{l}\text { gastric } \\
\text { cancer }\end{array}$ & Billroth II & 10 years & $\begin{array}{l}20 \mathrm{~cm} \text { more anal from } \\
\text { Braun's anastomotic region }\end{array}$ & $\begin{array}{l}\text { retro- } \\
\text { grade }\end{array}$ & resection \\
\hline 15 & $\begin{array}{l}\text { Ietsugu } \\
{[23]}\end{array}$ & 2001 & $72 / \mathrm{M}$ & $\begin{array}{l}\text { gastric } \\
\text { cancer }\end{array}$ & double tract & 1.5 year & $\begin{array}{l}10 \mathrm{~cm} \text { more anal from } \\
\text { jejuno-jejunal anastomotic } \\
\text { region }\end{array}$ & $\begin{array}{l}\text { retro- } \\
\text { grade }\end{array}$ & resection \\
\hline$\overline{16}$ & $\begin{array}{l}\text { Tahara } \\
{[24]}\end{array}$ & 2003 & $75 / \mathrm{F}$ & $\begin{array}{l}\text { plasma } \\
\text { cytoma }\end{array}$ & $\begin{array}{l}\text { Graham } \\
\text { method }\end{array}$ & 7 years & $\begin{array}{l}10 \mathrm{~cm} \text { more anal from } \\
\text { Braun's anastomotic region }\end{array}$ & $\begin{array}{l}\text { retro- } \\
\text { grade }\end{array}$ & ction \\
\hline 17 & $\begin{array}{l}\text { Akiyama } \\
{[25]}\end{array}$ & 2005 & $60 / \mathrm{M}$ & $\begin{array}{l}\text { gastric } \\
\text { cancer }\end{array}$ & Roux-en-Y & 4 years & $\begin{array}{l}10 \mathrm{~cm} \text { more anal from } \mathrm{R}-\mathrm{Y} \\
\text { anastomotic region }\end{array}$ & $\begin{array}{l}\text { retro- } \\
\text { grade }\end{array}$ & $\begin{array}{l}\text { reintegra- } \\
\text { tion }\end{array}$ \\
\hline 18 & $\begin{array}{l}\text { Matsumoto } \\
{[26]}\end{array}$ & 2005 & $74 / \mathrm{F}$ & $\begin{array}{l}\text { gastric } \\
\text { cancer }\end{array}$ & Roux-en-Y & 12 years & $\begin{array}{l}5 \mathrm{~cm} \text { more anal from } \mathrm{R}-\mathrm{Y} \\
\text { anastomotic region }\end{array}$ & $\begin{array}{l}\text { retro- } \\
\text { grade }\end{array}$ & resection \\
\hline 19 & $\begin{array}{l}\text { Sato } \\
{[27]}\end{array}$ & 2006 & $74 / \mathrm{F}$ & $\begin{array}{l}\text { gastric } \\
\text { cancer }\end{array}$ & Roux-en-Y & 21 years & $\begin{array}{l}20 \mathrm{~cm} \text { more anal from } \mathrm{R}-\mathrm{Y} \\
\text { anastomotic region }\end{array}$ & $\begin{array}{l}\text { retro- } \\
\text { grade }\end{array}$ & $\begin{array}{l}\text { reintegra- } \\
\text { tion }\end{array}$ \\
\hline 20 & our case & 2008 & $80 / \mathrm{F}$ & $\begin{array}{l}\text { gastric } \\
\text { cancer }\end{array}$ & Billroth II & 14 years & $\begin{array}{l}10 \mathrm{~cm} \text { more anal from } \\
\text { Braun's anastomotic region }\end{array}$ & $\begin{array}{l}\text { retro- } \\
\text { grade }\end{array}$ & resection \\
\hline
\end{tabular}

Fig. 1. a Preoperative abdominal X-ray indicates a localized expansion of the small intestine (arrow). b Abdominal CT indicates a lamellar structure arranged in a concentric circle (arrow). However, no mass lesion was observed.
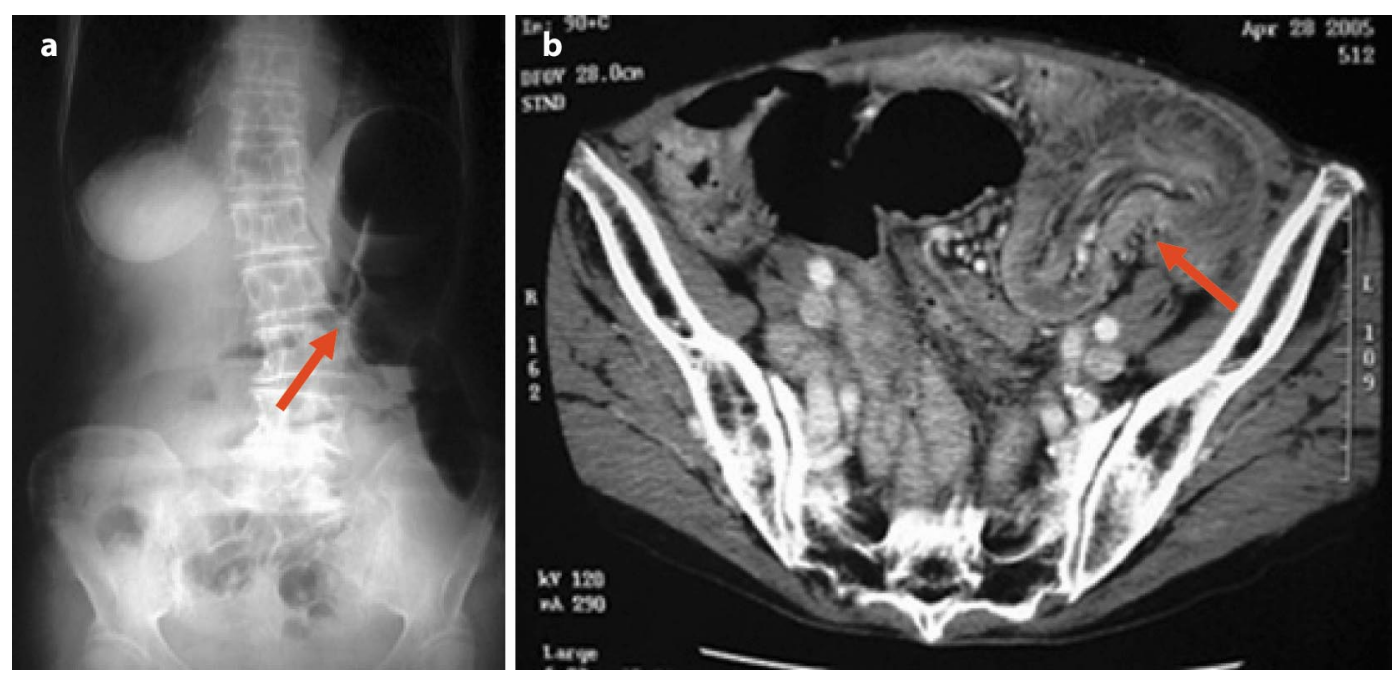
Fig. 2. The surgical findings of retrograde intussusception observed close to the anal side of the jejunojejunostomy.

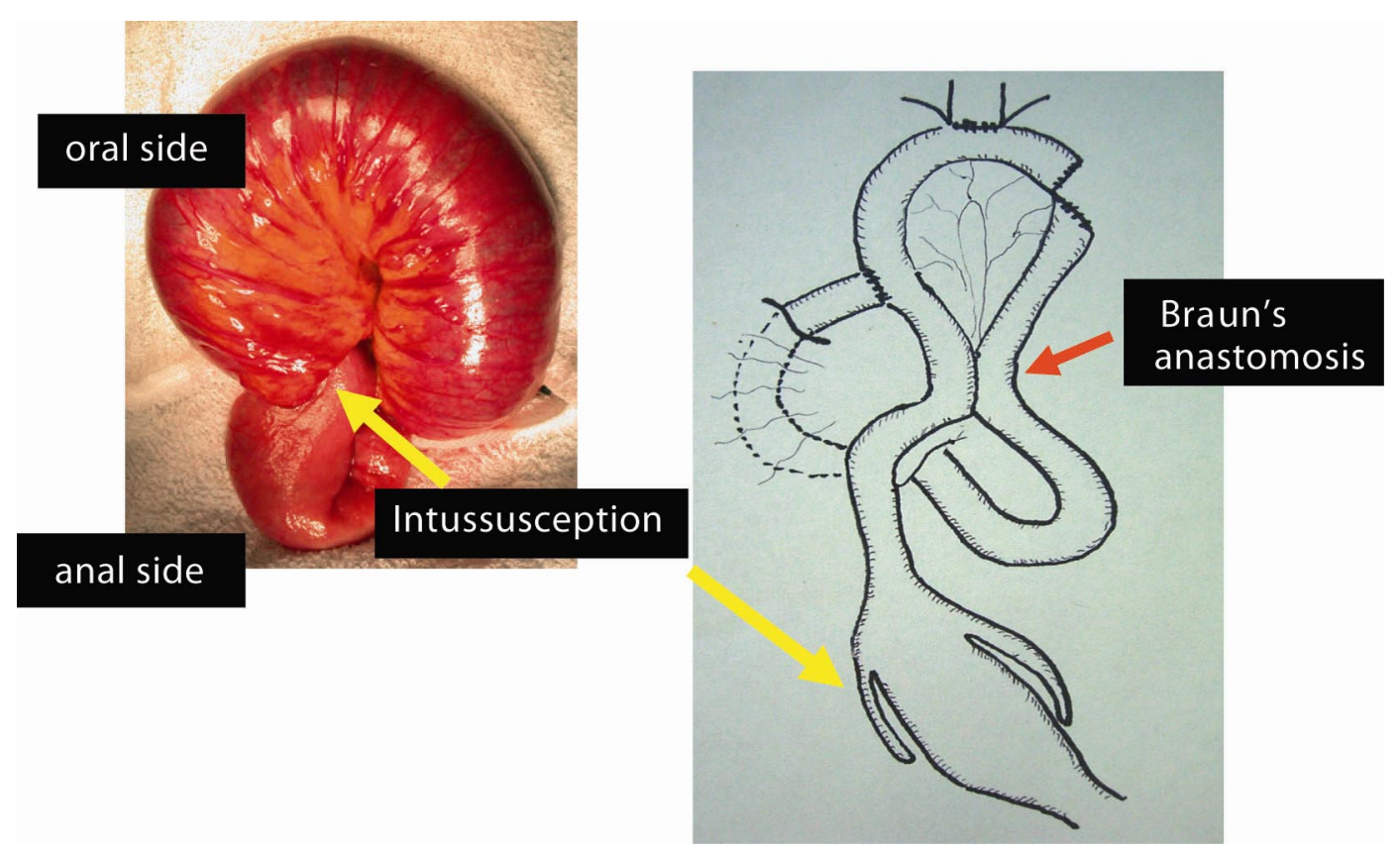




\section{References}

1 Narita H, Funabashi K, Yoshitomi H, Yamamori N, Iguchi T, Hori K, Hato M: Post operative intussusception - report of a case, and a comparison between adult and pediatric intussusceptions after laparotomy. Nihon Rinsyo Gekagekaigakukaishi 1991;52:2125-2131.

2 Maki T, Matsuo K, Ikehara Y, Yoshioka S, Oishi J, Tomita A: A case report of intussusception at Braun's anastomosis occurring 15 years after gastrectomy. Nihon Rinsyo Gekagaigakukaishi 2004;65:2392-2395.

-3 Mason LB, Williams RW, Marshburn ET: Retrograde jejunogastric intussusception following gastrectomy. Arch Surg 1960;81:485-491.

- 4 Olsen AK, Bo O: Intussusception as a complication of partial gastrectomy. A case report. Acta Chir Scand 1978;144:405-408.

5 Wheatley MJ: Jejunogastric intussusception diagnosis and management. J Clin Gastroenterol 1989;11:452-454.

6 Denath FM, Kweka EL: Retrograde intussusception of the bypassed duodenojejunal segment after Roux-en-Y gastrectomy: computed tomography findings. Can Assoc Radiol J 1991;42:135-138.

7 Jang WI, Kim ND, Bae SW, Kim WT, Kwon SO, Yoon KS, Kim SY: Intussusception into the enteroanastomosis after Billroth II gastric resection; diagnosed by gastroscopy. J Korean Med Sci 1989;4:51-54.

8 Starling JR, Croom RD 3rd: Acute jejunogastric intussusception. Am Surg 1976;42:778-781.

9 Vink M: Retrograde intussusception of the efferent jejunal loop after gastrectomy. Arch Chir Neerl 1950;2:377-384.

-10 Freeman FJ, Bernatz PE, Brown PW Jr: Retrograde intussusception after total gastrectomy. Report of a case. Arch Surg 1966;93:586-588.

11 Davey WW: Retrograde intussusception following Roux-loop anastomosis in total gastrectomy. Br J Surg 1954;42:102-103.

12 Kato M, Nakamura S, Hashimoto I: A case of intussusception of the jejunal afferent loop after a total gastrectomy. Geka Chiryo 1965;12:495-497.

13 Christeas N, Sfinias G: Retrograde jejuno-jejunal intussusception: a rare postgastrectomy complication. Br J Clin Pract 1968;22:439-441.

14 Hanyu N, Suzuki H, Miho O, Nagao F: A case of jejunal intussusception after total gastrectomy. Nihon Shokakigekagakkaishi 1984;17:791-793.

15 Ogata M, Kuroki T: A case report of intussusception at Braun's anastomosis occurring after total gastrectomy. Nippon Rinsyo Gekaigakukaishi 1988;49:860864.

16 Hwang CJ, Cheng JP, Wu CC, Fang TH, Wu TC, Liu TJ: Retrograde jejunojejunal intussusception. A rare complication following total gastric resection. Gaoxiong Yi Xue Ke Xue Za Zhi 1990;6:264-267.

17 Suganuma H, Kawamura Y, Mizumoto K, Kishi K, Kato K: A case of jejunal intussusception after total gastrectomy. Geka Shinryo 1992;5:673-676.

18 Hashimoto N, Fukano M, Sue K, Koyanagi N, Minagawa S: Adult retrograde intussusception after total gastrectomy. Geka 1993;55:828-830.

19 Narishima Y, Kobayashi N, Kuroda F: A case of retrograde intussusception of jejunum after total gastrectomy. Nihon Fukubu Kyukyuigakukaishi 1994;14:363365.

20 Goto Y, Yodonawa S, Hirano M, Fujiwara A, Ogawa I: Retrograde jejunal intussusception after total gastrectomy. Nippon Rinsyo Gekaigakukaishi 1999;61:1474-1477.

21 Yoshioka T, Kaneko A, Okamoto K, Iwado M, Yoshida H, Sakamoto M: A case of jejunal intussusception in an adult which occurred 11 years after total gastrectomy. Nippon Rinsyo Gekaigakukaishi 1999;60:1833-1836.

22 Sumi K, Ashida K, Oka A, Murata Y, Kinugasa Y, Hamazoe R, Miura N: A case of invagination into the Braun's anastomosis occurred 10 years after total gastrectomy. Shimane Igakukaishi 2000;4:203-207.

23 Ietsugu K, Sakatoku M, Kosugi M, Terahata S: A preoperatively diagnosed retrograde intissusception after total gastrectomy. Hokur J Surg 2001;20:41-44. 
24 Tahara H, Kuroda Y, Kuranishi F, OkamotoY, Toyota K, Nakahara M: Intussusception of the jejunal afferent loop developed 7 years after a total gastrectomy. Nippon Rinsyo Gekaigakukaishi 2003;64:1389-1393.

25 Akiyama Y, Aoki K, Nakaya T, Fujiwara H: A case of retrograde jejunal intussusception of Roux-en-Y anastomotic site after total gastrectomy. Geka 2005;67:587-589.

26 Matsumoto T, Kawamoto K, Sano K, Ogasahara K: Retrograde intussusception of the jejunal afferent loop developed 12 years after total gastrectomy. Kurashiki Tyuou Byoin Nenpo 2005;67:107-110.

27 Sato S, Shinoda M, Kawaguchi S, Abe M, Kunori T, Shinya F: Case report of 21year postgastrectomy retrograde intussusception of the jejunum occurring distal to a Roux-en-Y anastomosis. Nihon Fukubu Kyukyuigakukaishi 2006;26:465-467. 